\title{
Distance Aware Collision Avoidance Protocol for Ad-Hoc Underwater Acoustic Sensor Networks
}

\author{
Borja Peleato and Milica Stojanovic
}

\begin{abstract}
This paper proposes a channel access protocol for ad-hoc underwater acoustic networks which are characterized by long propagation delays and unequal transmit/receive power requirements. The protocol saves transmission energy by avoiding collisions while maximizing throughput. It is based on minimizing the duration of a hand-shake by taking advantage of the receiver's tolerance to interference when the two nodes are closer than the maximal transmission range. Nodes do not need to be synchronized, can move, are half-duplex, and use the same transmission power. This protocol achieves a throughput several times higher than that of the Slotted FAMA, while offering similar savings in energy. Although carrier sensing ALOHA offers a higher throughput, it wastes much more power on collisions.
\end{abstract}

Index Terms-Underwater acoustic networks, medium access control, collision avoidance, delay tolerance.

\section{INTRODUCTION}

W ITH the advances in acoustic modem technology, research in underwater communications today is focusing on the design of first wireless networks [1]. Challenges of the underwater acoustic environment include a severely limited bandwidth (typically a few $\mathrm{kHz}$ over several kilometers), poor quality of the physical link, and high latency caused by the low speed of propagation $(c=1500 \mathrm{~m} / \mathrm{s}$ is the nominal speed of sound underwater). In addition, the currently available acoustic modems operate in half-duplex, and the power required for transmitting is much greater than that required for receiving. As a result, direct application of the existing medium access control (MAC) design principles often results in an inefficient use of system resources. Rather than modifying the existing protocols, we take a different approach and design a protocol that takes advantage of the long propagation delays and signal absorption to maximize throughput.

The main source of energy consumption for an acoustic modem is, by far, transmitting (tens of Watts are typically required for transmission, with the exact number depending on the transmission distance, while reception requires tens of $\mathrm{mW}$ or up to a few $\mathrm{W}$, depending on the type of processing used [2]). Therefore, it is important to avoid packet collisions at the receiver when long data packets are being sent. This fact has been recognized recently by several authors, resulting in the design of MAC protocols that are particularly suited to the underwater acoustic channel: while [3] and [4] focus on sleep scheduling, [5] and [6] propose hand-shaking procedures

Manuscript received July 11, 2007. The associate editor coordinating the review of this letter and approving it for publication was Dr. Fabrizio Granelli.

Borja Peleato is with Stanford University (e-mail: peleato@stanford.edu, borjapeleato@yahoo.es).

Milica Stojanovic is with the Massachusetts Institute of Technology (email: millitsa@mit.edu).

Digital Object Identifier 10.1109/LCOMM.2007.071160. that guarantee absence of collisions. However, these protocols require long hand-shakes and some level of synchronization between the nodes. By setting transmission parameters in accordance with the maximal propagation delay, they lengthen the hand-shake procedure beyond the minimum needed. Such an approach is inefficient for those links that are much shorter than the transmission range.

Here we propose under the name of Distance-Aware Collision Avoidance Protocol (DACAP) a non-synchronized protocol that allows a node to use different hand-shake lengths for different receivers so as to minimize the average handshake duration. This is achieved by taking advantage of both the greater received power over short links, and the estimated distance between the nodes.

\section{Protocol Description}

The protocol is specified as follows. Upon receiving a request-to-send (RTS), a node (receiver) immediately replies with a clear-to-send (CTS), then waits for the data packet. If, after sending the CTS, it overhears a packet threatening its pending reception, the receiver sends a very short warning packet to its partner (the node to whom it had sent the CTS).

Upon receiving a CTS, a node (sender) waits some time before transmitting the data packet. If it overhears a packet meant for some other node or receives a warning from its partner, the node defers its transmission. In some cases the warning arrives while the node is transmitting the data, and hence is lost because modems are half duplex. However, the length of the idle period has been designed so that this only happens when the SIR is high enough to carry on the transmission. The length of the idle period will depend upon the distance between the nodes $U$, which the sender can learn by measuring the RTS/CTS round-trip time $2 U / c$.

The waiting period is chosen such as to guarantee absence of harmful collisions. With constant transmission power for all the nodes, not all the collisions are harmful because a packet coming from a far-away node is attenuated and it does not corrupt another packet coming from a node that is much closer. Hand-shakes thus only need to avoid collisions from nodes closer than a certain distance. Hence, hand-shakes between close neighbors can be made short, while those between farapart nodes need to be longer.

To achieve a trade-off that maximizes the throughput of a given network, a minimum hand-shake length $t_{\min }$ is predefined for all the nodes. For a network in which most links are close to the transmission range, $t_{\min }$ needs to be nearly $2 T$, twice the maximum propagation delay. When some links are shorter, it can be reduced. 
Two versions of the protocol have been designed: with acknowledgments sent right after receiving the data packet, and without acknowledgments.

\section{A. Case without acknowledgments}

When no acknowledgments are used, the receiver only sends a warning if it overhears an RTS within $2 T-t_{\min }$ seconds after sending a CTS, and the sender only defers the transmission if it receives a warning from its partner, or overhears a CTS within the next $t_{\min }$ seconds after sending an RTS.

Letting $U+\Delta D$ be the minimal distance to an interfering node for which correct reception is still possible, and $t_{\text {data }}$ the duration of the data packet to be transmitted, the waiting period is determined as

$$
T_{w}(U / c)= \begin{cases}t_{\text {min }}-2 U / c, & U / c<t_{1} \\ 2(U+\Delta D) / c-t_{\text {min }}, & U / c>t_{1}\end{cases}
$$

where

$$
t_{1}=\frac{t_{\text {min }}-\min \left(\Delta D / c, t_{\text {data }}, 2 T-t_{\text {min }}\right)}{2}
$$

and there is an additional restriction that $T_{w}>2 \Delta D / c$.

\section{B. Case with acknowledgments}

To accommodate this case, two new parameters are introduced: $\Delta t_{\text {data }}$, the maximum difference between the duration of two data packets, and $T_{W \_m i n}$, a predetermined minimum $T_{w}$. Additionally, $t_{\min }$ should be chosen greater than $\min \left(T, 2 \max _{U}(\Delta D / c)+\Delta t_{d a t a}\right)$ and $T_{W_{-} \text {min }}$ shorter than $\min \left(T, t_{\min }\right)$.

The receiver sends a warning when it overhears a CTS within $2 T-T_{W \_m i n}$, or an RTS within $2 T-t_{\min }$ seconds after transmitting a CTS. The sender defers the transmission if it receives such a warning or overhears either a CTS within $t_{\min }$, or an RTS within $T$ seconds after sending the RTS.

The waiting time is now given by

$T_{w}(U / c)= \begin{cases}2(U+\Delta D) / c-t_{\text {min }}, & U / c \in\left(t_{1}, t_{2}\right) \\ 2(U+\Delta D) / c-T_{W \_ \text {min }} & U / c>\max \left(t_{2}, t_{3}\right) \\ t_{\text {min }}-2 U / c & \text { other }\end{cases}$

where

$$
\begin{aligned}
t_{1} & =\frac{t_{\text {min }}-\min \left(\Delta D / c, t_{\text {data }}, 2 T-t_{\text {min }}\right)}{2} \\
t_{2} & =\frac{t_{\text {min }}-\Delta t_{\text {data }}}{2} \\
t_{3} & =\min \left(t_{1}, \frac{t_{\text {min }}+T_{W \_ \text {min }}-2 \Delta D / c}{4}\right)
\end{aligned}
$$

The additional restriction is expressed as $T_{W}(U / c)>$ $\max \left(2 \Delta D / c, T_{W \_ \text {min }}\right)$.

\section{BACK-OFFS}

When a node overhears an RTS or a CTS from a handshake going on within its transmission range, it goes into a back-off state to avoid interfering. As the position of the nodes performing the hand-shake is unknown, the back-off times are usually chosen longer than necessary. We propose two strategies to minimize the resulting inefficiencies:
- Nodes continue listening to the channel while in the backoff state, and return to the idle state if they detect the end of the current transmission (data packet or acknowledgment).

- If a node receives an RTS from one of the two nodes involved in the current transmission, it replies with a CTS and exits the back-off state.

This policy gives priority to the nodes already using the channel, making the protocol unfair. However, per-node fairness in sensor networks is usually of lesser importance that the overall information flow. Additionally, in the version with acknowledgments, priority is given to the receiver, thus making the way through the network smoother for packets that need multiple hops.

In order to prevent the system from falling into a deadlock, a random back-off delay has also been implemented after each unsuccessful hand-shake attempt.

\section{Simulation Results}

The simulation scenario was that of a $5 \mathrm{~km}$ by $5 \mathrm{~km}$ area divided into 16 squares with a node at a random location within each square. RTS and CTS packets are 48 bits long, warnings and acknowledgments are 24 bits long, and data packets have 9600 bits. The transmission rate is 4800 bps. Each node generates packets for random destinations according to a Poisson distribution, and has an infinite transmit queue. Minimum hop routing and noiseless channel are assumed.

The performance of the protocol was compared to that of Slotted FAMA [5], including Carrier Sensing ALOHA [7] as a benchmark. In CS-ALOHA, nodes transmit packets whenever they see the channel idle, and therefore do not waste time on hand-shaking. S-FAMA is a previously proposed protocol for the underwater environment, also based on RTS/CTS handshaking, that divides the time into slots whose length equals $T$. The packets are sent only at the beginning of a slot, which avoids all collisions regardless of their Signal to Interference Ratio (SIR).

To assess the protocol performance, we measure the throughput as a function of the offered load, defined as

$$
\begin{aligned}
\text { throughput } & =\frac{\# \text { end-to-end packets } \cdot t_{\text {data }}}{\text { simulation time }} \cdot \text { average \#hops } \\
\text { offered load } & =\frac{\# \text { generated packets } \cdot t_{\text {data }}}{\text { simulation time }} \cdot \text { average \#hops }
\end{aligned}
$$

We start by considering a transmission range of $7 \mathrm{~km}$ (fully connected network) and the protocol without acknowledgments. As most links are much shorter than $7 \mathrm{~km}$, we choose $t_{\text {min }}=T$. For the sake of simplicity, we assume $\Delta D=3.5 \mathrm{~km}$ for all the nodes and distances, which corresponds to $\mathrm{SIR} \geq 35$ $\mathrm{dB}$ in case of a collision. (The results for $\mathrm{SIR} \geq 20 \mathrm{~dB}$ can be found in [8]). Fig. 1 illustrates the results. DACAP and S-FAMA both waste about $2 \%$ of the power on collisions, but DACAP achieves a much higher throughput. CS-ALOHA offers higher throughput at low loads, but it wastes too much power on collisions $-20 \%$ when offered load is 0.5 , and $50 \%$ when offered load is 1 . Additionally, its throughput degrades as the load increases, which is not the case with either DACAP or S-FAMA. In the event of a load peak, DACAP gives priority to transmissions between close nodes, draining the load excess 


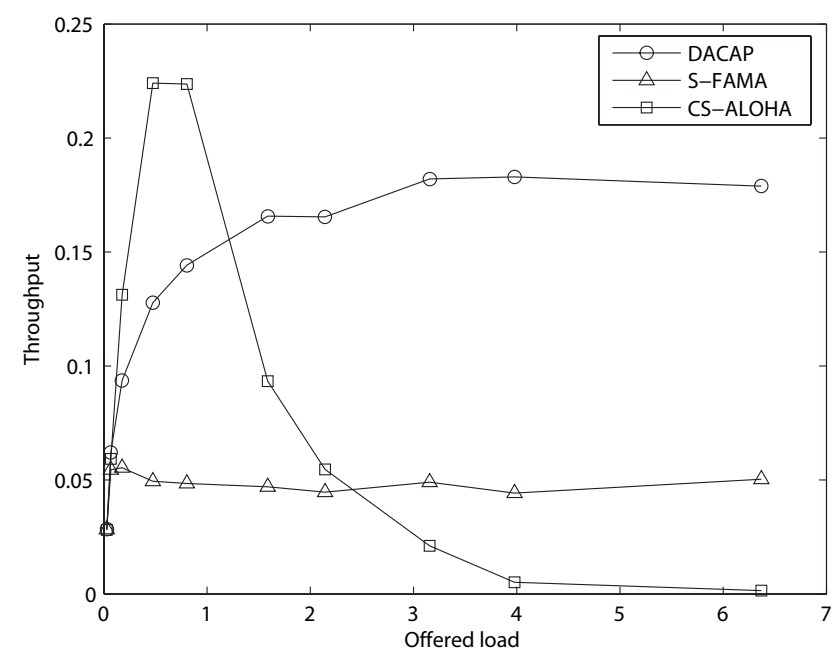

Fig. 1. Throughput of Carrier Sensing ALOHA, Slotted FAMA, and DACAP $\left(t_{\min }=T\right)$ without acknowledgments for a transmission range of $7 \mathrm{~km}$ and tolerance to interference given by $\Delta D=3.5 \mathrm{~km}$.

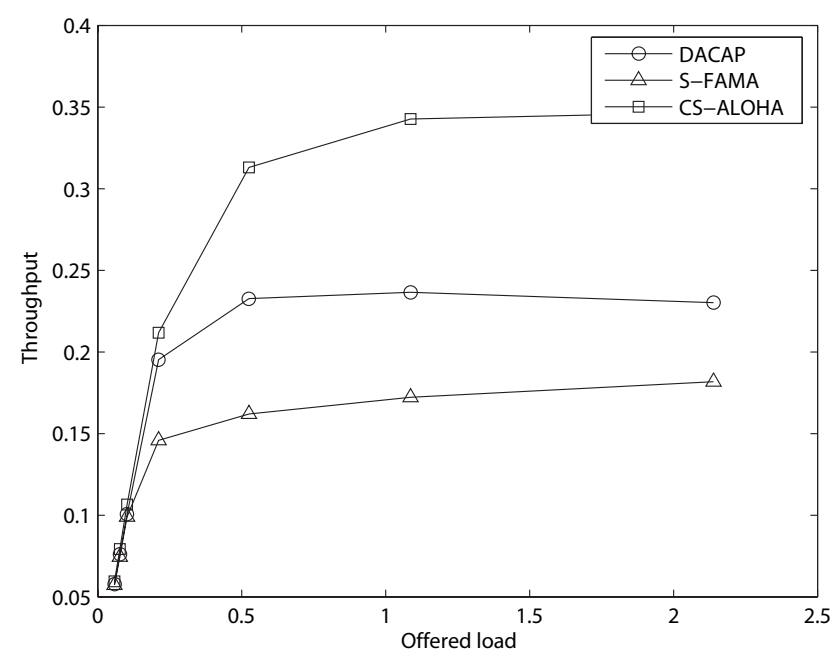

Fig. 2. Throughput of Carrier Sensing ALOHA, Slotted FAMA, and DACAP $\left(t_{\min }=2 T, T_{W \text { _min }}=0\right)$ with acknowledgments for a transmission range of $2.5 \mathrm{~km}$ and no tolerance to interference.

faster to return to the normal operation region (offered load between 0 and 1 ).

When acknowledgments are used, the protocols need to be slightly modified. CS-ALOHA introduces a $2 T$ seconds idle period after sending a data packet so as to wait for the acknowledgment. If it is not received, the node backsoff during a random period whose mean increases with each unsuccessful attempt [7]. As for S-FAMA, it is implemented as in [5], with the receiver sending the acknowledgment immediately after receiving the data and without using negative acknowledgments.

To illustrate the performance of the protocol with acknowledgments, we consider a transmission range of $2.5 \mathrm{~km}, t_{\min }=$ $2 T$, and $T_{W \_ \text {min }}=0$. An interesting case arises when $t_{\text {min }}=$ $2 T$ : the expressions for the waiting time (with and without acknowledgments) reduce to $T_{w}(U / c)=2 T-2 U / c$. Hence, all hand-shakes have the same duration regardless of the distance between the nodes. The advantage in this case is that no warnings are needed, but on the other hand, all handshakes are as long as with S-FAMA. Therefore, this design is most meaningful for networks with uniform transmitter-receiver distances on the order of the transmission range.

Fig. 2 shows the throughput when the nodes have no tolerance to interference $(\Delta D=2.5 \mathrm{~km}$, equal to the transmission range). Despite the addition of acknowledgments, higher throughput is achieved due to the shorter transmission range. CS-ALOHA is kept stable at high loads, but it still wastes too much power. The gap between DACAP and S-FAMA decreases because the former can no longer take advantage of the tolerance to interference. Hand-shakes are of equal duration for both, but DACAP achieves higher throughput because it concatenates transmissions without waiting for the beginning of the next slot.

\section{CONCLUSiON}

DACAP is a collision avoidance protocol that is easily scalable to the changing number of nodes and the coverage area of the network, and can be optimized for a particular network with very few constraints on the nodes. They do not need to share a common clock, can move, and the only parameters that they must locally share are $t_{\min }$ and $T$ (plus $T_{W \_ \text {min }}$ and $\Delta t_{\text {data }}$ if acknowledgments are required). Additionally, it provides higher throughput than Slotted FAMA with similar power efficiency. It is currently being considered for implementation in a real-time acoustic modem [9].

\section{ACKNOWLEDGMENT}

This work is supported in part by the National Science Foundation under Grants No. 0520075 and No. 0427502.

\section{REFERENCES}

[1] E. M.Sozer, M.Stojanovic, and J. G.Proakis, "Underwater acoustic networks," in IEEE J. Oceanic Eng., vol. 25, no. 1, Jan. 2000.

[2] L. Freitag, M. Grund, S. Singh, J. Partan, P. Koski, and K. Ball, "The WHOI micro-modem: an acoustic communications and navigation system for multiple platforms," in Proc. IEEE Oceans Conf. 2005.

[3] V. Rodoplu and M. K. Park, "An energy-efficient MAC protocol for underwater wireless acoustic networks," in Proc. IEEE Oceans Conf. 2005.

[4] W. Ye and J. Heidemann. "Ultra-low duty cycle MAC with scheduled channel polling," Tech. Report ISI-TR-604, USC/ISI, 2005.

[5] M. Molins and M. Stojanovic, "Slotted FAMA: a MAC protocol for underwater acoustic networks," in Proc. IEEE Oceans Conf. 2006.

[6] X. Guo, M. Frater, and M. Ryan, "A propagation-delay-tolerant collision avoidance protocol for underwater acoustic sensor networks," in Proc. IEEE Oceans Conf. 2006.

[7] S. Shahabudeen and M. Anil, "Design of networking protocols for shallow water peer-to-peer acoustic networks," in Proc. IEEE Oceans Conf. 2005.

[8] B. Peleato and M. Stojanovic, "A MAC protocol for ad hoc underwater acoustic sensor networks," in Proc ACM WUWNet 2006.

[9] E. M.Sozer and M. Stojanovic, "Reconfigurable acoustic modem for underwater sensor networks," in Proc ACM WUWNet 2006. 